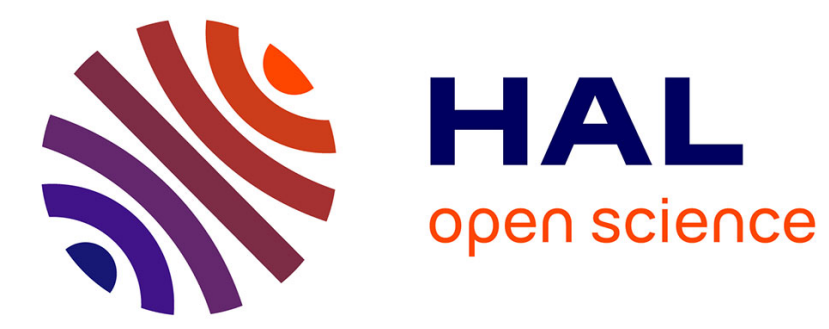

\title{
Piégeage des vortex
}

\author{
Y.A. Rocher, A. Némoz
}

\section{To cite this version:}

Y.A. Rocher, A. Némoz. Piégeage des vortex. Revue de Physique Appliquée, 1969, 4 (4), pp.579-580. 10.1051/rphysap:0196900404057900 . jpa-00243336

\section{HAL Id: jpa-00243336 https://hal.science/jpa-00243336}

Submitted on 1 Jan 1969

HAL is a multi-disciplinary open access archive for the deposit and dissemination of scientific research documents, whether they are published or not. The documents may come from teaching and research institutions in France or abroad, or from public or private research centers.
L'archive ouverte pluridisciplinaire HAL, est destinée au dépôt et à la diffusion de documents scientifiques de niveau recherche, publiés ou non, émanant des établissements d'enseignement et de recherche français ou étrangers, des laboratoires publics ou privés. 


\title{
PIÉGEAGE DES VORTEX
}

\author{
Y. A. ROCHER et A. NÉMOZ.
}

La séance commence par un rapide tour d'horizon des recherches actuelles effectuées et des techniques utilisées par les groupes présents :

C.R.T.B.T.-Grenoble (Nemoz). - Piégeage dans des alliages d'aluminium (Al-Ag, $\mathrm{Al}-\mathrm{Mg}$ ) dont la métallurgie est bien connue.

Techniques utilisées : mesures d'aimantation par intégration, mesure de température critique par résistivité. Investigation métallurgique par microscopie électronique et microdureté.

Alcatel (Rocher). - Piégeage des vortex dans le niobium en étudiant les caractéristiques $I(V)$ dans les régimes de flux-creep et de flux-flow.

Air Liquide (Renard). - Influence des inclusions ferromagnétiques dans les alliages plomb-thallium.

Techniques : caractéristiques $I(V)$ et mesures d'aimantation.

C.G.E. (Maldy). - Ancrage dans les couches minces de niobium. Effet de pic dans le plomb, le niobium et le niobium faiblement allié. Sauts de flux.

Techniques : mesure de résistivité et caractéristique $I_{\mathrm{c}}(H)$.

L.C.I.E. (Mailfert). - Étude d'alliages $\mathrm{Nb}-\mathrm{Zr}$, $\mathrm{Nb}-\mathrm{Ti}, \mathrm{Pb}-\mathrm{In}$.

Techniques : mesure de transition résistive, d'aimantation, hyperfréquence, atténuation ultrasonore, pénétration macroscopique du flux.

C.R.T.B.T.-Grenoble (Béthoux). — Étude d'alliages $\mathrm{Nb}-\mathrm{Hf}$ en fonction de la concentration, de l'écrouissage et des impuretés.

Technique : mesures d'aimantation.

E.N.S. (Thorel). - Étude sur des alliages Pb-In et $\mathrm{Nb}$ de grande pureté de la pénétration du flux en alternatif $(50$ à $30000 \mathrm{~Hz})$ et du transport d'entropie, détecté par un deuxième son dans l'hélium suprafluide $(300 \mathrm{~Hz})$.

C.N.E.T.-Lannion (Minet). - Déplacement de flux dans $\mathrm{Sn}, \mathrm{Pb}, \mathrm{Nb}$ par impulsions brèves en champ perpendiculaire.

C.R.T.B.T.-Grenoble (le Gilchrist). - Étude d'alliages $\mathrm{Pb}-\mathrm{In}$ et $\mathrm{Nb}-\mathrm{Ta}$ par variation de la résistance de surface en fonction de la fréquence; fréquence d'ancrage.

Orsay-Physique du solide (Rossier). - Relaxation nucléaire anormale dans les vortex (niobium).

C.E.N.-Saclay (Hlasnik). - Supraconducteurs en champ pulsé, influence des sauts de flux sur les pertes.

Laboratoire de physique théorique-Clermont-Ferrand ( $\mathrm{Fa}$ bre). - Dans des alliages $\mathrm{Nb}-\mathrm{Ti}$ et $\mathrm{Nb}-\mathrm{Zr}$, restauration de l'état normal à l'aide d'impulsions de champ, influence de la vitesse de l'impulsion sur la largeur de transition.

Technique utilisée : apparition de tension.

Laboratoire de physique du solide-Caen (Fortini). Étude de supraconducteurs de première espèce (plomb pur) par aimantation.

Orsay-I.U.T.-Ville d'Avray (Donadieu). - Dans des alliages niobium-titane et niobium-étain, sauts de flux macroscopiques par mesure de tension.

$\mathrm{Au}$ cours de la discussion, on s'intéresse essentiellement aux mécanismes du piégeage des vortex (nature des centres, répartition, effet de surface...) à l'exclusion des phénomènes liés aux sauts de flux.

La première intervention de Renard a trait à l'étude de l'influence des inclusions d'impuretés ferromagnétiques (Ni) dans un alliage de deuxième espèce, facile à fabriquer sans hystérésis, de $\mathrm{Pb}$ - $\mathrm{Tl}$. Les grains de $\mathrm{Ni}$ sont monodomaines; leur taille varie entre 200 et $600 \AA$ avec des concentrations de 60 à 100 ppm, donc une distance entre grains comprise entre $350 \AA(\simeq \xi)$ et $1200 \AA(\simeq \lambda)$. Le piégeage est dû à la disparition locale de la supraconductivité au voisinage des grains ferromagnétiques. Dans ces conditions, on peut exprimer la force d'ancrage $F_{\mathrm{A}}$ sous forme d'une loi réduite :

$$
\begin{gathered}
F_{\mathrm{A}}=f^{2}\left(1-t^{2}\right)^{2} \sim H_{\mathrm{c}}^{2} f^{2} \\
f^{2} \sim 1-\frac{B}{H_{\mathrm{c}_{2}}}
\end{gathered}
$$

Si l'on fait grossir les grains par recuit, la force d'ancrage diminue et on ne peut plus faire apparaître de loi réduite.

Ces inclusions n'ont aucune influence sur la température critique. A une question de Brochier deman- 
dant si cette force est clairement liée à la nature ferromagnétique des inclusions, C. Caroli rappelle l'article théorique de Heinrich qui calcule la déformation du paramètre d'ordre due à une impureté ferromagnétique. Si l'impureté n'est pas ferromagnétique, l'effet est extrêmement faible.

Maldy (C.G.E.) intervient ensuite, ses expériences portent sur l'ancrage des vortex existant dans des couches minces de $\mathrm{Pb}(\xi=1000 \AA)$ en champ perpendiculaire. L'observation en microscopie électronique ne montre que des joints de grains et des défauts de surface importants liés à l'existence de ces joints de grains, et peu de défauts du type inclusion ou dislocation.

Les résultats expérimentaux indiquent qu'au-delà d'un certain champ faible le courant critique obéit à la loi :

$$
J_{\mathrm{c}}\left(H+H_{0}\right)=\alpha_{\mathrm{c}}
$$

où $\alpha_{c}$ est proportionnel à la force d'ancrage et varie avec l'épaisseur du film, les forces d'ancrage sont de l'ordre de $10^{7}$ dynes $/ \mathrm{cm}^{3}$. L'explication proposée est basée sur le piégeage du vortex par les défauts de surface entraînant une courbure de ce vortex, qui ne peut dépasser une valeur critique. Un bilan des forces agissant sur ce vortex piégé, force d'ancrage et pression exercée par les autres vortex supposés libres dans les grains, et sa comparaison avec les résultats, conduit à ne faire intervenir qu'un nombre « efficace » de vortex libres évalué à $60 \%$ (grosses réserves de J. Matricon).

Une autre expérience sur des rubans épais de niobium $(30 \mu)$ met en évidence l'effet de pic d'une part, et la sensibilité aux états de surface d'autre part (polissage chimique et stries parallèles sur la surface).

Les défauts dont Rocher (Alcatel) parle dans la troisième intervention sont des dislocations. Les études ont porté sur du $\mathrm{Nb}$ laminé, dégazé sous ultravide et sur des monocristaux de $\mathrm{Nb}$ déformés à $4,2{ }^{\circ} \mathrm{K}$. Les résultats sont rapportés aux énergies d'ancrage selon le modèle de Baixeras-Fournet. Ces énergies d'ancrage sont très reproductibles d'un matériau à l'autre. Lorsqu'il n'y a pas d'effet de pic, ces énergies d'ancrage décroissent avec $B$. Lorsqu'il y a effet de pic, elles sont à peu près constantes hors de la région du pic. Dans tous les cas, elles décroissent quand la température diminue.

Les études ont porté aussi sur la variation du paramètre $\delta^{\mathbf{4}}$ de la théorie $\mathrm{du}$ 《flux-creep » $\left(V \sim \exp -\frac{E_{\mathrm{p}}-J B \delta^{4}}{k T}\right)$ en fonction de l'induction appliquée. Ce paramètre croît à peu près linéairement avec $B$, sauf dans la région de pic où il présente une anomalie. Il semble que les faibles valeurs de $I_{\mathrm{c}}$ dans les échantillons déformés soient dues à une limitation des forces bloquant les dislocations du réseau de vortex.

L'intervention suivante de Bethoux concernant l'étude des alliages $\mathrm{Nb}-\mathrm{Ta}$ et $\mathrm{Nb}-\mathrm{Hf}$ de concentration variable et à état d'écrouissage constant est plutôt du ressort de la table ronde « Matériaux ». La nature des centres de piégeage n'est pas abordée, mais les résultats montrent que les forces de piégeage croissent avec $B$ dans des alliages à moins de $50 \%$ Hf et décroissent avec $B$ pour des concentrations supérieures à $50 \%$.

Dans la discussion qui suit entre théoriciens et expérimentateurs, on retient les remarques suivantes :

- Les valeurs d'énergie de piégeage semblent prouver qu'elles sont plus caractéristiques du réseau de tourbillon que de la structure des défauts.

- A une question relative à l'intérêt d'étudier théoriquement le mouvement des dislocations $\mathrm{du}$ réseau de vortex plutôt que le mouvement d'un vortex, Matricon répond qu'en fait un vortex a plus de chances d'être piégé qu'une dislocation du réseau qui est une région où la densité de vortex est plus faible, donc où le paramètre d'ordre supraconducteur est plus homogène, et que d'autre part le mouvement d'une dislocation du réseau de vortex n'est que la résultante du mouvement individuel des vortex.

- Il apparaît nécessaire de dresser des tableaux de résultats nombreux et systématiques pour des matériaux ayant des défauts connus (force d'ancrage par unité de volume, courant critique en fonction du champ...), ce qui permettrait alors aux théoriciens d'estimer les facteurs importants et de bâtir ainsi un début de théorie.

- Il y a aussi intérêt, pour une plus grande efficacité, à ce que les métallurgistes et les physiciens se concertent (choix des matériaux, traitements thermiques...).

- Deux directions possibles d'étude se dégagent :

- Recherche de matériaux auto-stabilisants
$\quad\left(\frac{\mathrm{d} J_{\mathrm{c}}}{\mathrm{d} T}>0\right)$ en exploitant peut-être l'effet de pic;
- Visualisation du piégeage des vortex sur des défauts ou des surfaces à l'aide, par exemple, de la technique mise au point par Trauble et Essmann. 